\title{
A Possible Stream of Accreted Globular Clusters in M 31
}

\author{
H.-B. Yuan ${ }^{1,2}$ \\ ${ }^{1}$ Kavli Institute for Astronomy and Astrophysics, Peking University, Beijing 100871, China \\ email: yuanhb4861@pku.edu.cn \\ ${ }^{2}$ LAMOST Fellow
}

\begin{abstract}
Globular clusters (GCs) are excellent tracers for the formation, assembly and evolutionary history of their host galaxies. However, their origin and their role in galaxy evolution are still unclear. There are accumulating evidences that a significant fraction of GCs in massive galaxies (e.g., M 31) are accreted during their assembly history (e.g., Mackey et al. 2010). In this contribution, we report the discovery of a possible stream of accreted GCs in M 31 using data from the literature. Unlike previous substructures of GCs identified as clumps in the phase and metallicity space (Ashman, Keith \& Christina 1993), the members of this stream are widely spread but tightly correlated in the position-velocity space (see Fig. 1). The tight correlation suggests that they possibly follow very similar orbits. A number of stellar streams have been discovered in the outer halo of M 31 (e.g., Ibata et al. 2001; McConnachie et al. 2009), one of which may be physically associated with the GC stream. If the association is established, it will not only provide a key evidence for accretion origins of some GCs in M 31, but also place a strong constraint on the mass distribution of M 31 .
\end{abstract}

Keywords. galaxies: individual (M 31), globular clusters: individual (B 077, B 125, B 213, B 399, B 403, B 407, SH 25, EXT 8)
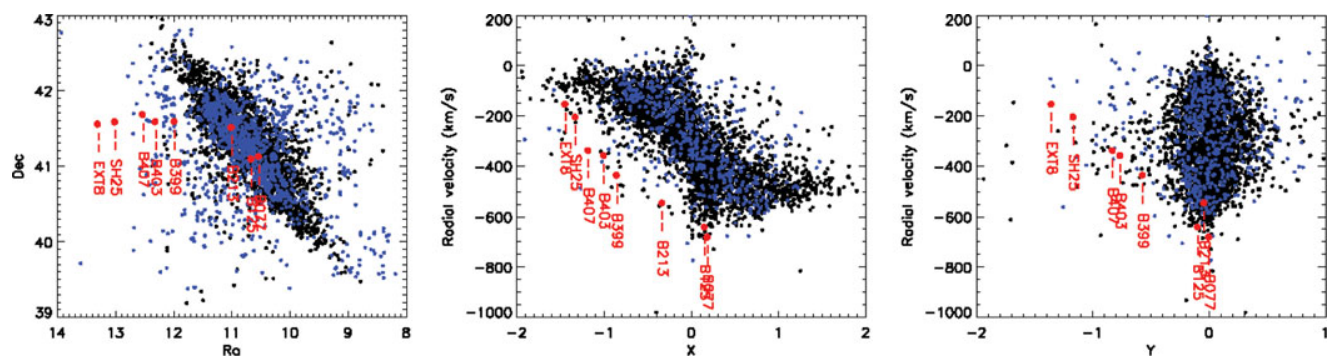

Figure 1. Spatial distribution (left panel) and position-velocity diagrams (middle and right panels) of the accreted GC stream in M 31. Black crosses: planetary nebulae from Merrett et al. (2006); Blue crosses: confirmed/candidate GCs from Revised Bologna Catalog V4.0 (Galleti et al. 2009); Red dots: members of the GC stream. The IDs of the GC members are also marked.

\section{References}

Ashman, K. M. \& Bird, C. M. 1993, AJ, 106, 2281

Galleti, S., Bellazzini, M., Buzzoni, A., Federici, L., \& Fusi Pecci, F. 2009, A\& $A$, 508, 1285

Ibata, R., Irwin, M., Lewis, G., Ferguson, A. M. N., \& Tanvir, N. 2001, Nature, 412, 49

Mackey, A. D., Huxor, A. P., Ferguson, A. M. N., et al. 2010, ApJL, 717, L11

McConnachie, A. W., Irwin, M. J., Ibata, R. A., et al. 2009, Nature, 461, 66

Merrett, H. R., Merrifield, M. R., Douglas, N. G., et al. 2006, MNRAS, 369, 120 